\title{
Study on the Prevalence of Vascular Calcification in Different Types of Arteries and Influencing Factors in Maintenance Peritoneal Dialysis Patients
}

\author{
Qingyu Niu ${ }^{a}$ Huiping Zhao ${ }^{a}$ Bei Wu ${ }^{a}$ Shihming Tsai ${ }^{a}$ Jian $W^{b}$ \\ Meng Zhang ${ }^{b}$ Lixia Lu $^{\text {a }}$ Jie Qiao ${ }^{a}$ Chuncui Men ${ }^{a}$ Li Zuo $^{a}$ Mei Wanga \\ aDepartment of Nephrology, Peking University People's Hospital, Beijing, China; \\ ${ }^{b}$ Department of Radiology, Peking University People's Hospital, Beijing, China
}

\section{Keywords}

Peritoneal dialysis · Vascular calcification · X-ray film ·

Abdominal aorta calcification

\begin{abstract}
Objective: To investigate the occurrence of vascular calcification (VC) in different types of arteries in patients with maintenance peritoneal dialysis (PD) patients and its influencing factors. Methods: This study enrolled PD patients with stable status who has received PD treatment for more than 6 months in Peking University People's Hospital. We used plain X-ray films of abdomen, pelvis, and hands to quantitatively evaluate VC of large artery (abdominal aorta, iliac artery), medium artery (femoral artery, radial artery), and small artery (finger arteries). Two radiologists read and scored radiographs blindly. Demographic data, clinical characteristics, Charlson comorbidity index (CCI), baseline and time-average laboratory indices including parameters of calcium phosphorus metabolism, serum albumin, PD adequacy were collected. A logistic regression model was used to estimate the influencing factors of different sites of VC. Results: (1) 154 PD patients were enrolled in this study: seventy-eight males, mean age was $60.4 \pm 13.9$ years, and median PD duration was 24 (16.39) months. The major primary disease was
\end{abstract}

diabetic nephropathy (39\%). (2) Among the 154 PD patients, the proportion of calcification of large artery was the highest (found in 100 patients, accounting for 64.9\%); then the medium artery $(66,42.9 \%)$; and 15 of small artery, accounting for $9.7 \%$. (3) Logistic regression showed that older age, longer dialysis duration, lower baseline serum intact parathyroid hormone (iPTH), and higher $\mathrm{CCl}$ scores were independent risk factors of large artery calcification $(p<0.05)$, and higher $\mathrm{CCl}$ scores, higher baseline serum triglycerides (TG), lower baseline serum iPTH, and time-average iPTH were independent risk factors of medium and small arteries. Conclusions: In PD patients, the occurrence of large artery calcification was higher than others. Among different sites of VC, the abdominal aortic calcification was most likely to occur, and the proportion of small artery calcification was low. Calcification of medium and small arteries can exist alone without calcification of large artery. Large artery calcification was more likely to occur in patients with older age, longer dialysis duration, lower baseline serum iPTH levels and higher $\mathrm{CCI}$ scores. Patients with higher CCl scores, higher baseline TG and lower baseline iPTH, and time-average iPTH were more likely to develop small and medium artery calcification.

C 2019 The Author(s)

Published by S. Karger AG, Basel

\begin{tabular}{ll}
\hline KARGER & $\begin{array}{l}\text { C } 2019 \text { The Author(s) } \\
\text { Published by S. Karger AG, Basel }\end{array}$ \\
E-Mail karger@karger.com & This article is licensed under the Creative Commons Attribution- \\
www.karger.com/bpu & $\begin{array}{l}\text { NonCommercial-NoDerivatives 4.0 International License (CC BY- } \\
\text { NC-ND) (http://www.karger.com/Services/OpenAccessLicense). } \\
\text { Usage and distribution for commercial purposes as well as any dis- } \\
\text { tribution of modified material requires written permission. }\end{array}$
\end{tabular}




\section{Introduction}

The incidence of cardiovascular disease (CVD) in patients with end-stage renal disease (ESRD) is 20-30 times higher than that of the general population [1]. Peritoneal dialysis (PD) is one of the main renal replacement treatments in ESRD patients. Registration data from different countries showed that CVD is the primary cause of mortality in PD patients, accounting for more than $50 \%$ of the causes of death [1-4]. Vascular calcification (VC) is a common complication of ESRD patients, an important cause of CVD, and an independent predictor of all-cause death and cardiovascular $(\mathrm{CV})$ death in dialysis patients $[5,6] . \mathrm{VC}$ is an important component of chronic kidney disease-mineral and bone disorder (CKD-MBD), which is a pathologic process that occurs in response to dysregulated or inappropriate environmental stimuli [7]. There are 2 main forms of arterial calcification in ESRD patients: intimal calcification, associated with atherosclerosis with irregular patchy calcification, and medial calcification, associated with aging, diabetes, and ESRD, commonly occurring in the peripheral muscular arteries with linear or orbital calcification [8].

The clinical practice guideline for CKD-MBD of Kidney Disease: Improving Global Outcomes (KDIGO) suggested that if patients with CKD stages 3-5 have vascular or valvular calcification, they should be seen as having the highest $\mathrm{CV}$ risk, and use this information to guide the management of CKD-MBD $[9,10]$. Therefore, it is important to estimate the occurrence of $\mathrm{VC}$ in $\mathrm{PD}$ patients and find out its influencing factors.

Regarding methods of assessing VC, electron beam computed tomography or multi-slice computed tomography are highly sensitive and specific for assessing the calcification score of coronary artery, and are the gold standard for diagnosing VC. However, due to the high demand for equipment and the high cost of inspection, they have been restricted as routine screening methods.

Although the prevalence of $\mathrm{VC}$ is higher in patients with CKD, arteries in some sites are not sensitive to calcification compared to large arteries, such as finger arteries [11]. The speed, mechanism, pathophysiological manifestations, risk factors, and prognosis of different grades of arterial calcification may be different. Most studies only described VC at specific sites: studies by Okuno et al. [5], Honkanen et al. [12], and Wong et al. [13] have reported significant associations between abdominal aortic calcification (AAC) and CV mortality and all-cause mortality in patients; Hong et al. [6] reported that calcification of iliac artery and femoral artery were associated with patients' all-cause mortality in a retrospective cohort study of 214 hemodialysis (HD) patients by using pelvic plain film. The vascular smooth muscle cells with different embryonic origin may have different calcification susceptibilities, and this may lead to different prevalence of VC in different sites of arteries [14]. O'Neill et al. [15] estimated breast artery calcification in CKD patients. Their study was based on histological analysis of small arteries and arterioles in breast tissue with diameter of $10-1,500 \mathrm{~mm}$. They concluded that the pathogenesis of medial calcification differs among different types and sizes of arteries. Furthermore, the expression and activity of calcification inhibitors such as matrix Gla protein and pyrophosphate in different blood vessels may vary and contribute to different susceptibility of VC. Adragao et al. [16] found that a simple VC score evaluated by plain radiographic films of pelvis and hands was strongly associated with $\mathrm{CV}$ mortality of $\mathrm{HD}$ patients.

Till now, most studies only described VC at specific sites, and rarely described VC in different sites of arteries in patients with dialysis [6]. There was no report on the occurrence of VC in different arteries of PD patients. The incidence of arterial calcification varied from site to site and the influencing factors may vary. Therefore, this study aimed to investigate the occurrence of $\mathrm{VC}$ in different sites of arteries (including abdominal aorta, iliac artery, femoral artery, radial artery, and finger artery) in PD patients by X-ray films. Meanwhile, we investigate the influencing factors of VC in different arteries to guide the treatment of complications and improve patients' prognosis.

\section{Materials and Methods}

\section{Study Design and Subjects}

This single-center cross-sectional observational study enrolled maintenance PD patients with ESRD in Peking University People's Hospital from April 2014 to August 2015. Inclusion criteria for participants were age $>18$ years old, PD duration $\geq 6$ months and stable clinical condition. Exclusion criteria were patients with acute complications such as heart failure, severe infection, and malignant tumors. The study was approved by the Ethics Committee of Peking UniversityPeople's Hospital(ethicalapprovalnumber:2018PHB073).

All the subjects used conventional glucose-based, lactate-buffered PD solutions (Ultrabag; Baxter Healthcare, Guangzhou, China; $\mathrm{Mg}^{2+} 0.25 \mathrm{mmol} / \mathrm{L}, \mathrm{Ca}^{2+} 1.25 \mathrm{mmol} / \mathrm{L}$ or $1.75 \mathrm{mmol} / \mathrm{L}, \mathrm{Na}^{+}$ $132 \mathrm{mmol} / \mathrm{L}$, and $\mathrm{Cl}^{-} 96 \mathrm{mmol} / \mathrm{L}$ ). The daily dialysate exchange dose was more than $6 \mathrm{~L}$, received either by continuous ambulatory $\mathrm{PD}$ or intermittent PD.

\section{Methods}

Demographic Data

Demographic information and clinical characteristics were collected for all enrolled patients. Data included age, gender, 
PD duration, primary renal disease, the presence or absence of diabetes, body mass index, and Charlson comorbidity index (CCI).

\section{Evaluation of VC}

We used lateral abdominal radiograph, frontal pelvic radiograph, and both hands radiographs to evaluate the calcification of abdominal aorta, iliac artery, femoral artery, radial artery, and finger arteries. According to the definition of anatomy and histology, the arteries were divided into large, medium, and small arteries according to the size of the luminal diameter and the components of the media $[17,18]$. The abdominal aorta and the iliac artery belong to the large artery, the femoral artery and the iliac artery belong to medium artery, and the finger arteries belong to the small artery.

The method used to evaluate calcification was described previously [19]: the lateral abdominal radiographs were divided into 2 sections by a horizontal line over the intervertebral space between L2 and L3; radiographs of the pelvis were divided by 2 lines: a horizontal line just above the femoral heads and a median vertical line; and radiographs of each hand were divided by a horizontal line over the proximal end of the metacarpals. The presence of calcification in any section was given 1 point and scores from all sectors summed up to a total score. The highest total score is 10 points. The radiographs were reviewed by 2 radiologists who were blinded to the real situation.

Baseline and Time-Average Laboratory Indices

The baseline clinical data were the values of laboratory indices within 3 months after starting PD treatment, including serum corrected total calcium $(\mathrm{Ca})$, phosphate $(\mathrm{P})$, serum intact parathyroid hormone (iPTH), alkaline phosphatase, albumin, triglycerides (TG), total cholesterol, serum creatinine, low-density lipoprotein cholesterol (LDL-C), carbon dioxide combining power, hemoglobin, total urea clearance, and total creatinine clearance rate.

The time average indices: laboratory indices were collected every 3 months from the start of PD to time of X-ray examination. After calculation, the arithmetic mean values were obtained as time-average indices. At the same time, the time-average systolic blood pressure, diastolic blood pressure, pulse pressure, PD ultrafiltration, and urine output were collected.

\section{Statistical Methods}

Measurement data are expressed as mean $\pm \mathrm{SD}$, or median with IQR appropriately, and categorical data are expressed as percentages or ratios. Differences in mean and median values between groups with and without AAC were evaluated by using the independent sample $t$ test or Wilcoxon rank-sum test respectively. Categorical data between groups were compared by using the chisquare test. The independent influencing factors of VC were analyzed by logistic regression analysis. The SPSS (version 22.0) statistical software was used for statistical analyses and $p$ value $<$ 0.05 was considered to be statistically significant.

\section{Results}

Demographic Data and Clinical Characteristics

A total of 154 PD patients were included in the present study, including 78 males (50.6\%), with an average age of
$60.4 \pm 13.9$ years $(21-75)$ and a median dialysis duration of $24(16,39)$ months. Primary renal disease was predominantly diabetic nephropathy $(n=60,39.0 \%)$, followed by chronic glomerulonephritis $(n=58,37.7 \%)$, chronic tubulointerstitial nephropathy ( $n=17,11.0 \%)$, hypertensive renal disease $(n=17,11.0 \%)$, and others $(n=2,1.3 \%)$. There were 65 patients (42.2\%) with diabetes. The clinical and laboratory data of the patients are shown in Table 1.

\section{VC in Different Sites of Arteries in PD Patients}

Among the 154 patients, the proportion of large artery calcification (abdominal aorta and/or iliac artery) was the highest ( $n=100,64.9 \%$ ); followed by the medium artery calcification (femoral artery and/or radial artery; $n=66$, 42.9\%) and small artery calcification (finger arteries; $n=$ $15,9.7 \%)$. The prevalence and distribution of VC in different sites are shown in Tables 2 and 3.

\section{Influencing Factors of VC in Different Sites of Arteries}

The Occurrence and Influencing Factors of Large

Artery Calcification in PD Patients

Patients were divided into 2 groups according to the presence or absence of large artery calcification. Compared with the non-large artery calcification group, patients in the large artery calcification group exhibited older age, longer PD duration, higher proportion of diabetes and diabetic nephropathy, higher CCI scores, higher baseline TG, lower baseline iPTH, and lower baseline albumin $(p<0.05$; Tables 4,5$)$. Logistic regression analysis showed that older age, longer dialysis duration, lower baseline serum iPTH, and higher CCI scores were independent risk factors of large artery calcification $(p<0.05$; Table 6).

The Occurrence and Influencing Factors of Medium and Small Arteries Calcification in PD Patients

Patients were divided into 2 groups according to the presence or absence of medium and small arteries calcification. Compared with the non-calcification group, patients in the medium and small arteries calcification group manifested an older age, higher proportion of male, higher proportion of diabetes and diabetic nephropathy, higher CCI scores, higher time-average creatinine clearance rate, higher baseline TG level, lower DBP, lower baseline and time-average iPTH level, and lower time-average TG level ( $p<0.05$; Tables 7,8$)$. Logistic regression analysis showed higher CCI scores and higher baseline TG levels, and lower baseline and timeaverage iPTH were independent risk factors of medium and small arteries calcification $(p<0.05$; Table 9$)$.
Niu/Zhao/Wu/Tsai/Wu/Zhang/Lu/Qiao/ Men/Zuo/Wang 
Table 1. Demographic and clinical characteristics of all patients

\begin{tabular}{|c|c|}
\hline Variables & All patients $(n=154)$ \\
\hline Age, years & $60.41 \pm 13.88$ \\
\hline Gender, male, $n(\%)$ & $78(50.6)$ \\
\hline PD duration, months & $24(16-39)$ \\
\hline \multicolumn{2}{|l|}{ Primary renal disease, $n(\%)$} \\
\hline $\mathrm{DN}$ & $60(39.0)$ \\
\hline CGN & $58(37.7)$ \\
\hline CTIN & $17(11.0)$ \\
\hline Hypertensive renal disease & $17(11.0)$ \\
\hline Others & $2(1.3)$ \\
\hline \multicolumn{2}{|l|}{ Baseline laboratory indices } \\
\hline Serum corrected $\mathrm{Ca}, \mathrm{mmol} / \mathrm{L}$ & $2.31 \pm 0.24$ \\
\hline Serum $\mathrm{P}, \mathrm{mmol} / \mathrm{L}$ & $1.49 \pm 0.36$ \\
\hline Serum iPTH, pg/ml & $166.67 \pm 160.22$ \\
\hline Serum ALP, mmol/L & $83.56 \pm 42.45$ \\
\hline $\mathrm{HB}, \mathrm{g} / \mathrm{L}$ & $114.66 \pm 10.33$ \\
\hline Serum ALB, g/L & $38.40 \pm 3.88$ \\
\hline Serum TG, mmol/L & $1.89 \pm 0.84$ \\
\hline Serum Tcho, mmol/L & $4.97 \pm 1.04$ \\
\hline Serum $\mathrm{CO}_{2} \mathrm{CP}, \mathrm{mmol} / \mathrm{L}$ & $27.89 \pm 9.11$ \\
\hline Diabetes, $n(\%)$ & $65(42.2)$ \\
\hline SBP, mm Hg & $130.96 \pm 13.45$ \\
\hline $\mathrm{DBP}, \mathrm{mm} \mathrm{Hg}$ & $76.75 \pm 9.86$ \\
\hline Urinary output, $\mathrm{mL} / 24 \mathrm{~h}$ & $410(113-766.75)$ \\
\hline Total daily fluid removal, $\mathrm{mL}$ & $592(526.8-689.5)$ \\
\hline $\mathrm{BMI}, \mathrm{kg} / \mathrm{m}^{2}$ & $23.02 \pm 3.71$ \\
\hline $\mathrm{CCI}$ & $3(2-4)$ \\
\hline \multicolumn{2}{|l|}{ Time-average laboratory indices } \\
\hline Serum corrected $\mathrm{Ca}, \mathrm{mmol} / \mathrm{L}$ & $2.34 \pm 0.12$ \\
\hline Serum $\mathrm{P}, \mathrm{mmol} / \mathrm{L}$ & $1.49 \pm 0.23$ \\
\hline Serum iPTH, mmol/L & $197.09 \pm 131.56$ \\
\hline Serum ALP, mmol/L & $80.44 \pm 24.13$ \\
\hline $\mathrm{Kt} / \mathrm{V}$, /week & $1.68(1.63-1.72)$ \\
\hline Ccr, L/week/1.73 $\mathrm{m}^{2}$ & $50.34(48.66-52.79)$ \\
\hline $\mathrm{HB}, \mathrm{g} / \mathrm{L}$ & $113(109.8-116)$ \\
\hline Serum ALB, g/L & $38.42 \pm 2.89$ \\
\hline Serum Scr, umol/L & $790.56 \pm 228.74$ \\
\hline Serum TG, mmol/L & $1.99 \pm 0.65$ \\
\hline Serum Tcho, $\mathrm{mmol} / \mathrm{L}$ & $5.07 \pm 0.71$ \\
\hline $\mathrm{CO}_{2} \mathrm{CP}, \mathrm{mmol} / \mathrm{L}$ & $26.9(25.7-27.7)$ \\
\hline
\end{tabular}

DN, diabetic nephropathy; CGN, chronic glomerulonephritis; CTIN, chronic tubulointerstitial nephropathy; Ca, calcium; P, phosphate; iPTH, intact parathyroid hormone; ALP, alkaline phosphatase; $\mathrm{HB}$, hemoglobin; ALB, albumin; TG, triglycerides; $\mathrm{CO}_{2} \mathrm{CP}$, carbon dioxide combining power; $\mathrm{BMI}$, body mass index; $\mathrm{CCI}$, Charlson comorbidity index; Kt/V, total urea clearance; Ccr, creatinine clearance rate.

\section{Discussion}

According to the study of Gan et al. [19], the sensitivity and specificity of plain radiography in the diagnosis of abdominal aortic calcification is 60.2 and $100 \%$, respec-
Table 2. Vascular calcification in different sites of PD patients $(n=$ 154)

\begin{tabular}{llr}
\hline & $\begin{array}{l}\text { Presence, } \\
n(\%)\end{array}$ & \multicolumn{1}{c}{$\begin{array}{l}\text { Absence, } \\
n(\%)\end{array}$} \\
\hline Abdominal aortic calcification & $93(60.4)$ & $61(39.6)$ \\
Iliac artery calcification & $39(25.3)$ & $115(74.7)$ \\
Femoral artery calcification & $57(37.0)$ & $97(63.0)$ \\
Radial artery calcification & $38(24.7)$ & $116(75.3)$ \\
Digital artery calcification & $15(9.7)$ & $139(90.3)$ \\
\hline \multicolumn{1}{c}{ PD, peritoneal dialysis. } & & \\
\hline
\end{tabular}

Table 3. The distribution of vascular calcification in different sites of arteries $(n=111)$

\begin{tabular}{lllc}
\hline $\begin{array}{l}\text { Large artery } \\
\text { calcification }\end{array}$ & $\begin{array}{l}\text { Medium artery } \\
\text { calcification }\end{array}$ & $\begin{array}{l}\text { Small artery } \\
\text { calcification }\end{array}$ & $n(\%)$ \\
\hline+ & - & - & $45(40.5)$ \\
+ & + & - & $41(36.9)$ \\
+ & - & + & $0(0)$ \\
- & + & - & $10(9.0)$ \\
- & - & + & $0(0)$ \\
- & + & + & $1(0.9)$ \\
+ & + & + & $14(12.6)$ \\
\hline
\end{tabular}

tively. And the sensitivity and specificity of plain radiography in the diagnosis of iliac and femoral arteries calcification is 24.8 and $100 \%$, respectively [19]. Therefore, our study used X-ray plain films to estimated VC of different sites. We found that the large artery (abdominal aorta, iliac artery) had the highest calcification rate (64.9\%), and the calcification rate of the medium and small arteries (femoral artery, radial artery, and digital artery) was $42.9 \%$. The median calcification score was 3 (2.6) in patients with large artery calcification, and the median calcification score was 5 (3.7) in patients with calcification of the medium and small arteries, suggesting that the calcification was more severe in patients with medium and small arteries calcification; $83.3 \%$ of medium and small arteries calcification was accompanied by large artery calcification and $16.7 \%$ was not, indicating that calcification of medium and small arteries can also exist alone without large artery calcification. The incidence of calcification in different arteries is different and may be related to the structure of different size of arterial walls. Atherosclerosis often involves large arteries and promotes the formation of patchy calcification in the arterial intima. Calcification of 
Table 4. Comparisons of demographic and clinical data between patients with and without large artery calcification

\begin{tabular}{|c|c|c|c|c|}
\hline Age, years & $65.36 \pm 11.50$ & $51.24 \pm 13.30$ & -5.51 & $0.000^{*}$ \\
\hline Gender, male, $n(\%)$ & $52(52.0)$ & $26(48.2)$ & 0.208 & 0.736 \\
\hline $\mathrm{DM}, n(\%)$ & $56(56.0)$ & $9(16.7)$ & 22.24 & $0.000^{*}$ \\
\hline $\mathrm{SBP}, \mathrm{mm} \mathrm{Hg}$ & $132.37 \pm 13.39$ & $130.08 \pm 16.04$ & -0.944 & 0.347 \\
\hline DBP, mm Hg & $76.88 \pm 10.53$ & $76.69 \pm 9.45$ & -0.111 & 0.912 \\
\hline $\mathrm{PP}, \mathrm{mm} \mathrm{Hg}$ & $55.49 \pm 13.91$ & $53.39 \pm 14.09$ & 0.889 & 0.375 \\
\hline Total daily fluid removal, $\mathrm{mL}$ & $600.00(522.32-650.00)$ & $604.55(497.50-718.75)$ & -0.477 & 0.633 \\
\hline $\mathrm{BMI}, \mathrm{kg} / \mathrm{m}^{2}$ & $23.24 \pm 3.74$ & $22.55 \pm 3.64$ & -1.095 & 0.275 \\
\hline
\end{tabular}

$* p<0.05$.

$\mathrm{PD}$, peritoneal dialysis; CCI, Charlson comorbidity index; PP, pulse pressure; BMI, body mass index.

Table 5. Comparisons of biochemical data between patients with and without large artery calcification

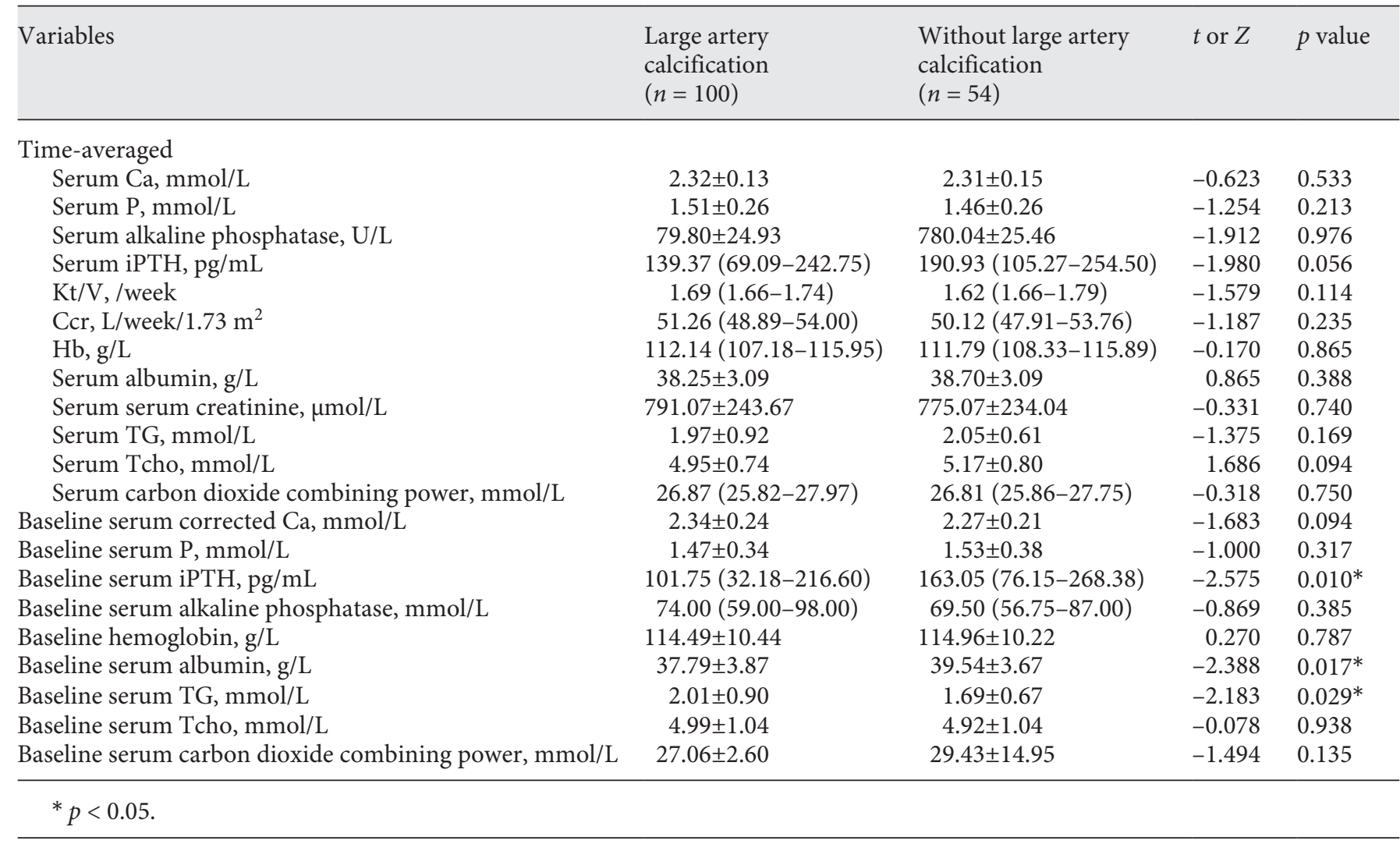


Table 6. Influencing factors independently associated with large artery calcification in PD patients in the logistic regression model

\begin{tabular}{lcccc}
\hline Variables & B & OR & 95\% CI & $p$ value \\
\hline Age, years & 0.081 & 1.084 & $1.045-1.124$ & $0.000^{*}$ \\
PD duration, months & 0.036 & 1.036 & $1.007-1.066$ & $0.013^{*}$ \\
CCI score & 0.504 & 1.656 & $1.123-2.440$ & $0.011^{*}$ \\
Baseline serum iPTH, pg/mL & -0.004 & 0.996 & $0.993-0.999$ & $0.016^{*}$ \\
\hline
\end{tabular}

$* p<0.05$.

$\mathrm{PD}$, peritoneal dialysis; CCI, Charlson comorbidity index; iPTH, intact parathyroid hormone.

Table 7. Comparisons of demographics and clinical data between patients with and without medium and small arteries calcification

\begin{tabular}{|c|c|c|c|c|}
\hline Variables & $\begin{array}{l}\text { Medium and small artery } \\
\text { calcification }(n=66)\end{array}$ & $\begin{array}{l}\text { Without medium and small } \\
\text { artery calcification }(n=88)\end{array}$ & $t$ or $Z$ & $p$ value \\
\hline Calcification score & $5.00(3.00-7.00)$ & $0(0-0)$ & -9.321 & $0.000^{*}$ \\
\hline Age, years & $63.85 \pm 12.43$ & $57.83 \pm 14.41$ & -2.778 & $0.005^{*}$ \\
\hline Gender, male, $n(\%)$ & $40(60.6)$ & $38(43.2)$ & 4.581 & $0.036^{*}$ \\
\hline PD duration, months & $24.0(15.0-35.0)$ & $24.0(16.0-42.8)$ & -0.698 & 0.485 \\
\hline $\mathrm{DN}, n(\%$ & $43(65.2)$ & $19(21.6)$ & 29.755 & $0.000^{*}$ \\
\hline $\mathrm{DM}, n(\%)$ & $44(66.7)$ & $21(23.9)$ & 28.327 & $0.000^{*}$ \\
\hline $\mathrm{SBP}, \mathrm{mm} \mathrm{Hg}$ & $132.26 \pm 14.49$ & $131.06 \pm 14.33$ & -0.512 & 0.609 \\
\hline $\mathrm{DBP}, \mathrm{mm} \mathrm{Hg}$ & $79.32 \pm 9.82$ & $74.94 \pm 10.01$ & -2.708 & $0.008^{*}$ \\
\hline $\mathrm{PP}, \mathrm{mm} \mathrm{Hg}$ & $52.94 \pm 14.88$ & $56.12 \pm 13.16$ & 1.401 & 0.163 \\
\hline Total daily fluid removal, mL & $602.28(432.50-634.37)$ & $602.28(536.65-700.00)$ & -1.524 & 0.127 \\
\hline $\mathrm{BMI}, \mathrm{kg} / \mathrm{m}^{2}$ & $23.55 \pm 3.86$ & $22.58 \pm 3.55$ & -1.501 & 0.133 \\
\hline CCI & $4.0(4.0-5.0)$ & $2.0(2.0-3.3)$ & -6.411 & $0.000^{*}$ \\
\hline
\end{tabular}

$* p<0.05$.

$\mathrm{PD}$, peritoneal dialysis; $\mathrm{BMI}$, body mass index; CCI, Charlson comorbidity index.

large arteries observed in plain films includes both intimal calcification associated with atherosclerosis and medial calcification. Meanwhile, medium and small arteries are less susceptible to atherosclerosis, so the calcification observed in the X-ray films is mainly the medial calcification. A study showed that in the linear calcification mechanism of small arteries, passive deposition of calcium salts is more effective than active ossification, which may be one of the reasons for the different rates of calcification in different sizes of arteries [6].

The results of Logistic regression analysis showed that older age, longer dialysis duration, higher CCI scores, and lower baseline serum iPTH were independent risk factors of large artery calcification. Aging is a traditional risk factor for VC. As the age increases, lipid deposition, increased connective tissue components, decreased smooth muscle and elastin, and calcification are observed in the intima of the vessel wall. However, long dialysis duration may cause the imbalance of positive regulators (such as inflammatory cytokines, reactived oxygen species, advanced glycation end products, $\mathrm{Ca}, \mathrm{P}, \mathrm{iPTH}$ ) and negative regulators (such as klotho, matrix-gla protein, fetuin-A, osteoprotegerin, and osteopontin) in uremia environment [20], and promote the occurrence of VC, which are consistent with studies reported $[21,22]$. Compared with large artery calcification, aging and dialysis duration have less effect on the medium and small arteries calcification. It may be that the body aging, tissue degradation, and atherosclerosis have less effect on the medium and small arteries calcification.

Considering that VC is a complication of slow onset and progression, time-average laboratory indices may better evaluate the control of patients with comorbidities and investigate the influencing factors of VC. Therefore, our study included time-average laboratory indices and baseline indices of PD patients. Previous studies have shown that high levels of serum $\mathrm{P}, \mathrm{Ca}$, and $\mathrm{PT} \mathrm{PH}$ were risk factors 
Table 8. Comparisons of biochemical data between patients with and without medium and small artery calcification

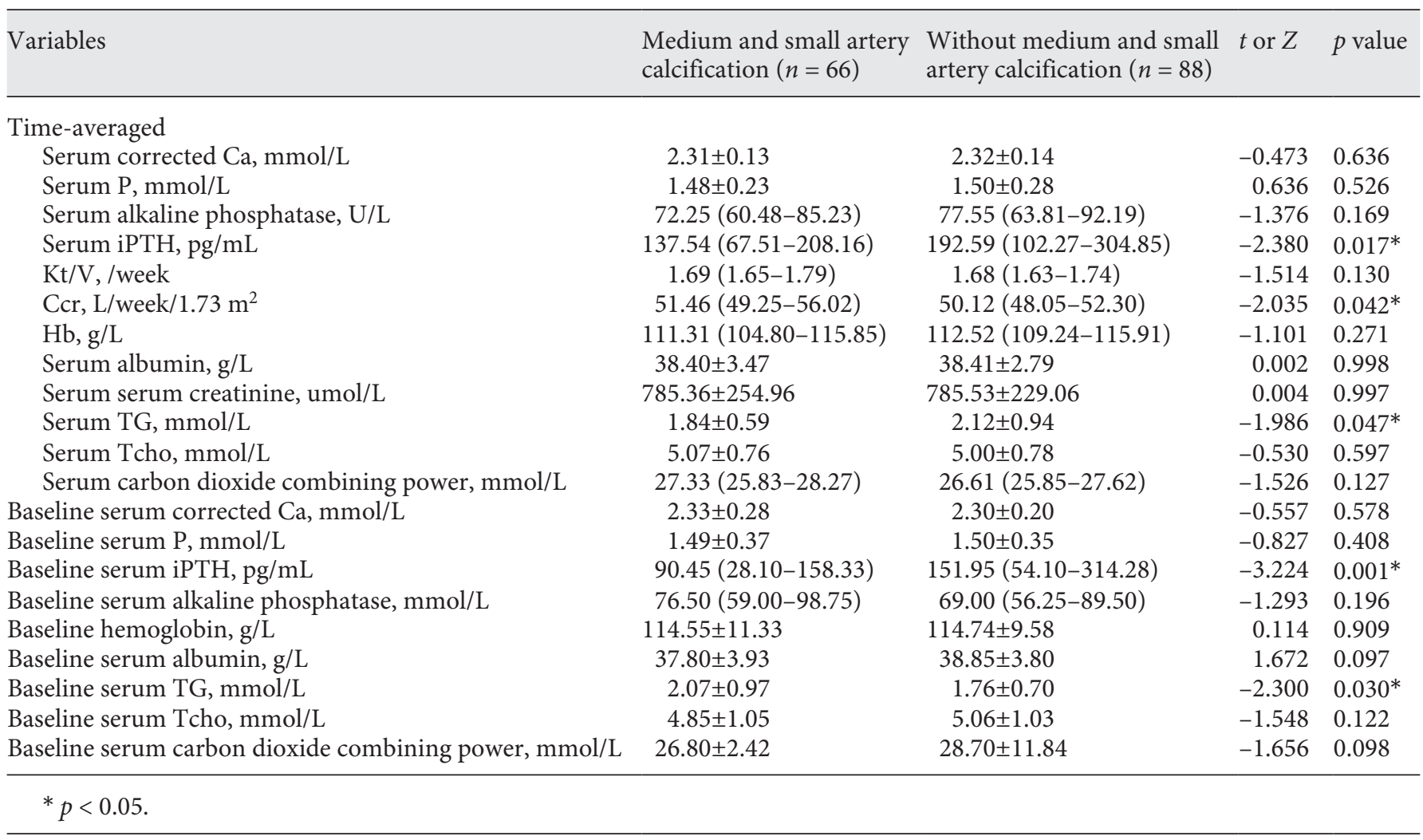

Table 9. Influencing factors independently associated with medium and small artery calcification in PD patients in the logistic regression model

\begin{tabular}{lcccc}
\hline Variables & B & OR & 95\% CI & $p$ value \\
\hline CCI score & 0.942 & 2.564 & $1.722-3.817$ & $0.000^{*}$ \\
Baseline serum iPTH, pg/mL & -0.005 & 0.995 & $0.991-0.999$ & $0.008^{*}$ \\
Time-average serum iPTH, pg/mL & -0.004 & 0.996 & $0.992-0.999$ & $0.026^{*}$ \\
Baseline serum TG, mmol/L & 0.726 & 2.068 & $1.136-3.764$ & $0.017^{*}$ \\
\hline
\end{tabular}

$* p<0.05$.

PD, peritoneal dialysis; CCI, Charlson comorbidity index; iPTH, intact parathyroid hormone; TG, triglycerides.

for VC in dialysis patients. IPTH can increase the expression of cartilage matrix and increase the intracellular calcium content, thereby promoting the formation of VC [21, 22]. The results of our study showed that there was no significant difference in serum $\mathrm{Ca}$ and $\mathrm{P}$ levels between patients with and without large artery calcification. There was no correlation between baseline and time-average serum $\mathrm{Ca}$, P level, and $\mathrm{VC}$, while lower serum $\mathrm{iPTH}$ was an independent influencing factor of larger, medium, and small arteries calcification. The possible reasons are as fol- lows: (1) our center has consistently adhered to continuous quality improvement for $\mathrm{Ca}$ and $\mathrm{P}$ metabolic disorders, so the laboratory indices of most patients are within the target range. The average serum $\mathrm{Ca}$ of the enrolled patients is 2.32 $\mathrm{mmol} / \mathrm{L}, \mathrm{P} 1.49 \mathrm{mmol} / \mathrm{L}$, which minimized the influence of Ca and P metabolic disorders on patients. The 2009 and 2017 KDIGO guidelines suggested that the target range of iPTH for patients with CKD stage $5 \mathrm{D}$ is approximately 2 to 9 times the upper normal limit for the assay $[9,10]$, for us, is $150-600 \mathrm{pg} / \mathrm{ml}$. The mean time-average $\mathrm{PTH}$ of the
14
Niu/Zhao/Wu/Tsai/Wu/Zhang/Lu/Qiao/ Men/Zuo/Wang 
enrolled patients in this study was $197.09 \mathrm{pg} / \mathrm{mL}$, of which $52(33.8 \%)$ were at $150-300 \mathrm{pg} / \mathrm{mL}, 34(22.1 \%)$ were at $300-600 \mathrm{pg} / \mathrm{mL}$, and $67(43.5 \%)$ were less than $150 \mathrm{pg} / \mathrm{mL}$. There were 58 (37.7\%) patients with baseline iPTH levels of $150-600 \mathrm{pg} / \mathrm{mL}, 91(59.1 \%)$ with $<150 \mathrm{pg} / \mathrm{mL}$, and few patients with high iPTH $(>600 \mathrm{pg} / \mathrm{mL})$, only $5(3.2 \%)$. Therefore, the relationship between high iPTH and VC cannot be confirmed, but it indicates that lower iPTH is also prone to VC. This result is consistent with recent studies: both high iPTH and low iPTH levels may cause VC [23, 24]. A study by London et al. [25] showed that HD patients with VC had lower iPTH levels than patients without VC. Elder et al. [26] reported that an HD patient with low bone turnover was treated by recombinant human PTH and bone biopsy demonstrated the mineral apposition rate increased, and serum and urine markers were consistent with improved bone turnover. These influences may have contributed to the resolution of calciphylaxis. Animal studies of Shao et al. [27] suggested that an action of intermittent injection of recombinant human PTH-(1-34) is a direct inhibition of VC and proliferation through actions on vascular PTH receptors. This reminds us not only to pay attention to the iPTH level when patients already starting $\mathrm{PD}$ treatment, but also to follow-up the CKD patients who have not started dialysis. Pay attention to the regular monitoring of iPTH before dialysis and rationally apply the active vitamin $\mathrm{D}$ to maintain the balance of $\mathrm{Ca}$ and $\mathrm{P}$ to avoid low iPTH and even low bone turnover in CKD patients. After starting dialysis treatment, it is necessary to maintain regular monitoring of iPTH level to avoid the occurrence and progress of VC. (3) The occurrence of VC is a complex process involving multiple factors. The Ca load (including drugs and dialysate), malnutrition, immune status, and aging are all influencing factors, which may affect the onset and progression of VC.

The present study showed that high CCI score was a common risk factor for large, medium, and small arteries calcification. Patients with complications at the beginning of PD were more likely to develop VC, indicating that VC was associated with comorbidities, especially $\mathrm{CKD}$ and diabetes. Therefore, patients who have various comorbidities at the beginning of dialysis should be actively treated for each comorbidity, and regular monitoring serum $\mathrm{Ca}$ and $\mathrm{P}$ levels and the progression of VC [25].

In addition to higher CCI scores and lower baseline and time-average iPTH levels, higher baseline TG was also an independent risk factor for medium and small arteries calcification. In CKD patients, the phenomenon of lipid metabolism disorder is quite common. Elevated TG levels accelerate lipid exchange and can cause elevated

Arterial Calcification in PD
LDL levels and decreased HDL levels. Chronic oxidative stress situation converts LDL into oxidized LDL. Oxidized LDL can induce vascular smooth muscle cells transdifferentiate into osteoblasts and accelerate the progression of VC. HDL plays a key role in maintaining vascular reactivity and anti-oxidative stress in endothelial cells, which can slow the progression of atherosclerosis.

There were also some limitations in this study. First, the number of study participants was relatively small. Second, the evaluation of the progression of VC in patients has not been considered; thus, the impact of progress rate of $\mathrm{VC}$ on prognosis may be ignored. Third, this study did not include the patient's medication, such as the use of calcium-containing P binders, calcitriol, and other drugs. These drugs may have impacts on the patient's Ca and $\mathrm{P}$ metabolism and VC.

\section{Conclusion}

In summary, in PD patients, the occurrence and influencing factors of different sites of arterial calcification were different. The proportion of large artery calcification was highest, and the proportion of small artery calcification was the lowest. Calcification of small and medium arteries can exist alone without calcification of large artery. Large artery calcification was more likely to occur in patients with older age, longer PD duration, lower baseline iPTH level, and higher CCI scores. Patients with higher CCI scores, higher baseline iPTH level, and higher baseline TG level were more likely to develop small and medium arteries calcification. Regular assessment of VC is beneficial to guide the clinical management and treatment of CKD-MBD.

\section{Ethics Statement}

The study was approved by the Ethics Committee of Peking University People's Hospital. As this was a retrospective study using medical records without any intervention, informed consent was exempted by the Ethics Committee.

\section{Disclosure Statement}

No conflict of interest is declared by any of the authors.

\section{Funding Source}

This research did not receive any specific grant from funding agencies in the public, commercial, or not-for-profit sectors. 


\section{References}

1 Wang AY, Brimble KS, Brunier G, Holt SG, Jha V, Johnson DW, et al: ISPD cardiovascular and metabolic guidelines in adult peritoneal dialysis patients part I - assessment and management of various cardiovascular risk factors. Perit Dial Int 2015;35:379-387.

2 Collins AJ, Foley R, Herzog C, Chavers B, Gilbertson D, Ishani A, et al: Excerpts from the United States Renal Data System 2007 annual data report. Am J Kidney Dis 2008;51(1 suppl 1):S1-S320.

3 de Jager DJ, Grootendorst DC, Jager KJ, van Dijk PC, Tomas LM, Ansell D, et al: Cardiovascular and noncardiovascular mortality among patients starting dialysis. JAMA 2009; 302:1782-1789.

4 Adequacy of dialysis and nutrition in continuous peritoneal dialysis: association with clinical outcomes. Canada-USA (CANUSA) Peritoneal Dialysis Study Group. J Am Soc Nephrol 1996;7:198-207.

5 Okuno S, Ishimura E, Kitatani K, Fujino Y, Kohno K, Maeno Y, et al: Presence of abdominal aortic calcification is significantly associated with all-cause and cardiovascular mortality in maintenance hemodialysis patients. Am J Kidney Dis 2007;49:417-425.

6 Hong D, Wu S, Pu L, Wang F, Wang J, Wang $Z$, et al: Abdominal aortic calcification is not superior over other vascular calcification in predicting mortality in hemodialysis patients: a retrospective observational study. BMC Nephrol 2013;14:120

7 Amann K: Media calcification and intima calcification are distinct entities in chronic kidney disease. Clin J Am Soc Nephrol 2008;3: 1599-1605.

8 Proudfoot D, Shanahan CM: Biology of calcification in vascular cells: intima versus media. Herz 2001;26:245-251.

9 Kidney Disease: Improving Global Outcomes (KDIGO) CKD-MBD Work Group: KDIGO clinical practice guideline for the diagnosis, evaluation, prevention, and treatment of chronic kidney disease-mineral and bone dis- order (CKD-MBD). Kidney Int Suppl 2009; 113:S1-S130.

10 Garabed E, Norbert L, Bertram LK; Kidney disease: Improving Global Outcomes (KDIGO) CKD-MBD Update Work Group: KDIGO 2017 clinical practice guideline update for the diagnosis, evaluation, prevention, and treatment of chronic kidney disease-mineral and bone disorder (CKD-MBD). Kidney Int Suppl 2017;7:S1-S59.

11 Noce A, Canale MP, Capria A, Rovella V, Tesauro M, Splendiani G, et al: Coronary artery calcifications predict long term cardiovascular events in non diabetic Caucasian hemodialysis patients. Aging (Albany NY) 2015;7: 269-279.

12 Honkanen E, Kauppila L, Wikstrom B, Rensma PL, Krzesinski JM, Aasarod K, et al: Abdominal aortic calcification in dialysis patients: results of the CORD study. Nephrol Dial Transplant 2008;23:4009-4015.

13 Wong ND, Lopez VA, Allison M, Detrano RC, Blumenthal RS, Folsom AR, et al: Abdominal aortic calcium and multi-site atherosclerosis: the multiethnic study of atherosclerosis. Atherosclerosis 2011;214:436-441.

14 Leroux-Berger M, Queguiner I, Maciel TT, Ho A, Relaix F, Kempf H: Pathologic calcification of adult vascular smooth muscle cells differs on their crest or mesodermal embryonic origin. J Bone Miner Res 2011;26:15431553.

15 O'Neill WC, Adams AL: Breast arterial calcification in chronic kidney disease: absence of smooth muscle apoptosis and osteogenic transdifferentiation. Kidney Int 2014;85:668676.

16 Adragao T, Pires A, Lucas C, Birne R, Magalhaes L, Goncalves M, et al: A simple vascular calcification score predicts cardiovascular risk in haemodialysis patients. Nephrol Dial Transplant 2004;19:1480-1488.

17 Tang JM, Zhang L: Histology and Embryology. Beijing, Peking University Medical Press, 2013.
18 Zhang CY: Human Anatomy. Beijing, People's Medical Publishing House, 1977, vol 1.

19 Gan LY, Wang M, Yu XY, et al: Application of plain radiography for diagnosis of vascular calcification in maintenance hemodialysis patients. Chin J Nephrol 2009;2:81-85.

20 Takeuchi K, Zhang B, Ideishi M, Urata H, Noda K, Tashiro T, et al: Influence of age and hypertension on the association between small artery compliance and coronary artery disease. Am J Hypertens 2004;17(12 pt 1): 1188-1191.

21 Cozzolino M, Mazzaferro S, Pugliese F, Brancaccio D: Vascular calcification and uremia: what do we know? Am J Nephrol 2008;28: 339-346.

22 Shroff RC, McNair R, Figg N, Skepper JN, Schurgers L, Gupta A, et al: Dialysis accelerates medial vascular calcification in part by triggering smooth muscle cell apoptosis. Circulation 2008;118:1748-1757.

23 Malluche HH, Porter DS, Monier-Faugere MC, Mawad H, Pienkowski D: Differences in bone quality in low- and high-turnover renal osteodystrophy. J Am Soc Nephrol 2012;23: 525-532.

24 Lu KC, Wu CC, Yen JF, Liu WC: Vascular calcification and renal bone disorders. Scientific World Journal 2014;2014:637065.

25 London GM, Marchais SJ, Guerin AP, Boutouyrie P, Metivier F, de Vernejoul MC: Association of bone activity, calcium load, aortic stiffness, and calcifications in ESRD. J Am Soc Nephrol 2008;19:1827-1835.

26 Elder G, Kumar KS: Calciphylaxis associated with chronic kidney disease and low bone turnover: management with recombinant human PTH-(1-34). NDT Plus 2008;1:9799.

27 Shao JS, Cheng SL, Charlton-Kachigian N, Loewy AP, Towler DA: Teriparatide (human parathyroid hormone (1-34)) inhibits osteogenic vascular calcification in diabetic low density lipoprotein receptor-deficient mice. J Biol Chem 2003:278:50195-50202. 